MATHEMATICS OF COMPUTATION

Volume 70, Number 235, Pages 897-909

S 0025-5718(01)01286-8

Article electronically published on March 7, 2001

\title{
ASYMPTOTICALLY EXACT A POSTERIORI ESTIMATORS FOR THE POINTWISE GRADIENT ERROR ON EACH ELEMENT IN IRREGULAR MESHES. PART 1: A SMOOTH PROBLEM AND GLOBALLY QUASI-UNIFORM MESHES
}

\author{
W. HOFFMANN, A. H. SCHATZ, L. B. WAHLBIN, AND G. WITTUM
}

\begin{abstract}
A class of a posteriori estimators is studied for the error in the maximum-norm of the gradient on single elements when the finite element method is used to approximate solutions of second order elliptic problems. The meshes are unstructured and, in particular, it is not assumed that there are any known superconvergent points. The estimators are based on averaging operators which are approximate gradients, "recovered gradients", which are then compared to the actual gradient of the approximation on each element. Conditions are given under which they are asympotically exact or equivalent estimators on each single element of the underlying meshes. Asymptotic exactness is accomplished by letting the approximate gradient operator average over domains that are large, in a controlled fashion to be detailed below, compared to the size of the elements.
\end{abstract}

\section{INTRODUCTION}

In this introduction we shall roughly describe our ideas and results. A precise statement of our results is given in Section 2 .

Consider a uniformly elliptic and coercive second order partial differential equation with homogeneous natural conormal Neumann boundary conditions on a bounded domain $\Omega$ in $R^{N}$. Let all data, i.e., $\partial \Omega$, coefficients, and the right-hand side be sufficiently smooth. In order to approximate the solution $u$, let $S_{h}=S_{h}^{r-1}(\Omega)$ be the simplicial $\mathcal{C}^{0}$ Lagrange elements of total polynomial degree $\leq r-1$ on exact partitions of $\Omega$ which are face-to-face and globally quasi-uniform, with curved faces allowed at $\partial \Omega$. Let $u_{h} \in S_{h}$ be the standard Galerkin approximation of $u$ based on the weak formulation (with all integrals done exactly). For technical reasons, we restrict ourselves to $r \geq 3$ in the present paper - the piecewise linear case is not included. The results do go over to the piecewise linear case, but the proof of this requires some new estimates not covered by Lemma 3.2 below. This will be the subject of a forthcoming investigation.

With $\tau$ denoting a typical simplex in a partition of $\Omega$, we shall consider a posteriori estimators $\mathcal{E}(\tau)$ for the local gradient error $\|\nabla e\|_{L_{\infty}(\tau)}, e=u-u_{h}$. Our estimators will be given in terms of approximate gradient operators $\mathcal{G}_{H} u_{h}, H \geq h$, where

Received by the editor November 20, 1998.

2000 Mathematics Subject Classification. Primary 65N30, 65N15.

The second and third authors were supported by the National Science Foundation, USA.

(C)2001 American Mathematical Society 
$\mathcal{G}_{H}$ takes into account the values of $u_{h}$ over a domain $d_{H} \supseteq \tau$, with $\operatorname{diam}\left(d_{H}\right)=H$. $\left(\mathcal{G}_{H} u_{h}\right.$ is sometimes referred to as a "recovered gradient".) It will be essential to our arguments to allow $H$ to be sufficiently large compared to $h$.

With finite element spaces as described, we can in general only expect that $\|\nabla e\|_{L_{\infty}(\tau)} \simeq O\left(h^{r-1}\right)$. A crucial property of the operator $\mathcal{G}_{H}$ is that it can approximate gradients of functions to "higher order", in the sense that

$$
\left\|\nabla v-\mathcal{G}_{H} v\right\|_{L_{\infty}\left(d_{H}\right)} \leq C_{\mathcal{G}} H^{r}\|v\|_{W_{\infty}^{r+1}\left(d_{H}\right)} \quad \text { for } v \in \mathcal{C}^{r+1}\left(\bar{d}_{H}\right) .
$$

Another crucial property we shall require is that

$$
\left\|\mathcal{G}_{H} v\right\|_{L_{\infty}\left(d_{H}\right)} \leq C_{\mathcal{G}} H^{-1}\|v\|_{L_{\infty}\left(d_{H}\right)} \quad \text { for } v \in W_{\infty}^{1}\left(\bar{d}_{H}\right)
$$

which may be thought of as an inverse, or smoothing, property. (Examples will be given below.) We then take our a posteriori error estimator over any single element $\tau \in d_{H}$ to be

$$
\mathcal{E}(\tau):=\left\|\nabla u_{h}-\mathcal{G}_{H} u_{h}\right\|_{L_{\infty}(\tau)} .
$$

Note that a fixed domain $d_{H}$ works for every element $\tau$ contained in it. It is thus not necessary to change the operator $\mathcal{G}_{H}$ for each and every element.

Our main result may be described as follows: Fix a parameter $\varepsilon, 0<\varepsilon<1$. There is then a constant $C^{\prime}$, independent of $h, H$ and $u$ (it depends on $\varepsilon$, on the differential operator, on $\Omega$, on the quasi-uniformity of the meshes, and on $C_{\mathcal{G}}$ ) such that one of the following two alternatives, I or II below, hold. In the first alternative, conditions are given for asymptotic exactness of $\mathcal{E}(\tau)$.

Alternative I. Suppose that on the element $\tau$, the function $u$ satisfies the "nondegeneracy" condition

$$
|u|_{W_{\infty}^{r}(\tau)} \geq h^{1-\varepsilon}\|u\|_{W_{\infty}^{r+1}(\Omega)} .
$$

In this case, with $m=C^{\prime}\left(h / H+(H / h)^{r} h^{\varepsilon}\right)$, it follows that

$$
\frac{1}{(1+m)} \mathcal{E}(\tau) \leq\|\nabla e\|_{L_{\infty}(\tau)},
$$

and, for $H$ and $h$ such that $m<1$,

$$
\frac{1}{(1+m)} \mathcal{E}(\tau) \leq\|\nabla e\|_{L_{\infty}(\tau)} \leq \frac{1}{(1-m)} \mathcal{E}(\tau) .
$$

If $m$ is bounded, we thus see that $\frac{1}{(1+m)} \mathcal{E}(\tau)$ furnishes a lower bound for the real gradient error on the single element $\tau$. If $m \leq \bar{m}<1$, as $h$ and $H$ vary, we call $\mathcal{E}(\tau)$ an equivalent estimator. This would be the case e.g. if $H=K h$ with $K$ fixed and sufficiently large (and $h$ sufficiently small). We call $\mathcal{E}(\tau)$ an asymptotically exact estimator if $m \rightarrow 0$ as $h \rightarrow 0$ so that $\mathcal{E}(\tau)$ is equivalent to $\|\nabla e\|_{L_{\infty}(\tau)}$ with a constant approaching 1 . This would be the case e.g. if $H=H(h)$ were chosen so that both $h / H \rightarrow 0$ and $(H / h) h^{\varepsilon / r} \rightarrow 0$ as $h \rightarrow 0$; roughly speaking, $H / h \rightarrow \infty$ but not too fast.

Alternative II. Suppose that (1.3) does not hold, i.e., $|u|_{W_{\infty}^{r}(\tau)}<h^{1-\varepsilon}\|u\|_{W_{\infty}^{r+1}(\Omega)}$. In this case,

$$
\|\nabla e\|_{L_{\infty}(\tau)}+\mathcal{E}(\tau) \leq\left(m+C^{\prime}\right) h^{r-\varepsilon}\|u\|_{W_{\infty}^{r+1}(\Omega)} .
$$

That is, if $m$ stays bounded, both the real gradient error on $\tau$ and our estimator are "superconvergent", $h^{r-\varepsilon} \ll h^{r-1}$, if $h$ is small enough. 
Note that if, for a subdomain $\Omega_{1} \subseteq \Omega$, we have

$$
\inf _{x \in \Omega_{1}} \sum_{|\alpha|=r}\left|D^{\alpha} u(x)\right| \geq c_{1}>0
$$

and if $\|u\|_{W_{\infty}^{r+1}(\Omega)}$ is bounded, then (1.3) holds for all $\tau \subseteq \Omega_{1}$, for $h$ sufficiently small.

The central technical tools used in proving the above results are the sharply localized a priori error estimates for $\|e\|_{L_{\infty}(\tau)}$ and $\|\nabla e\|_{L_{\infty}(\tau)}$ of Schatz $[9]$.

It may seem disappointing that we can accomplish asymptotic exactness only in the case of Alternative I. However, taking Alternative II to its extreme, it appears exceedingly hard to construct an a posteriori estimator that would detect if, by chance, $\nabla e \equiv 0$ on a single element. It also appears difficult to assess the error if its main part is due to pollution from outside the element. As will be seen (in (2.12) below), assumption (1.3) implies that the error $\|\nabla e\|_{L_{\infty}(\tau)}$ behaves essentially as the interpolation error on the element, and it is thus free from pollution. In a future publication we will give results analogous to the present ones for rough problems involving (controlled) pollution.

We shall now give three examples of approximate gradient operators $\mathcal{G}_{H}$.

Example 1.1. Let $d_{H} \subseteq \Omega$ be any shape-regular simplex of diameter $H$, and let $I_{H}^{r}$ denote interpolation into $\Pi^{r}\left(d_{H}\right)$, the polynomials of total degree $\leq r$ on $d_{H}$, at the principal lattice points. Note that $r$ is one degree higher than $r-1$, the polynomial degree of $S_{h}$. Set $\mathcal{G}_{H} u_{h}=\nabla I_{H}^{r} u_{h}$. If the basic mesh is done by systematic refinements of coarser meshes, it may be possible to take $d_{H}$ as a union of simplices $\tau$.

Since $I_{H}^{r} u_{h}$ is a polynomial, taking its gradient may be thought of as forming (complicated) difference quotients of its values at the principal lattice points. Since these lattice points are spaced $O(H)$ apart, for $H \gg h, \mathcal{G}_{H} u_{h}$ may be interpreted as a finite-element oriented "compact" way of forming sufficiently "long" and "accurate" difference quotients of $u_{h}$. Our results are also valid for classical $O\left(H^{r}\right)$-accurate difference quotients on lines, which may be taken to be one-sided close to $\partial \Omega$. In practice, from the point of view of additional programming, it may be more convenient to form such classical difference quotients rather than to compute with polynomials not originally present in the computer code.

Finally, since

$$
\frac{v(x+H)-v(x-H)}{2 H}=\frac{1}{2 H} \int_{x-H}^{x+H} v^{\prime}(t) d t=\frac{d}{d x}\left(\frac{1}{2 H} \int_{x-H}^{x+H} v(t) d t\right),
$$

we see that forming difference quotients is an averaging operator on the gradient or, equivalently, the derivative of an averaging operator on the function.

Example 1.2. Let $d_{H} \subseteq \Omega$ be such that $d_{H}$ contains a ball $\underline{B}$ of radius $\underline{c}_{1} H, \underline{c}_{1}>0$, is contained in a concentric ball $\bar{B}$ of radius $\bar{c}_{1} H$, has surface measure $\leq C_{2} H^{N-1}$, and is such that $\bar{B} \cap \Omega$ is star-shaped with respect to a point $\bar{x}$. In particular, $d_{H}$ could be a mesh domain. With $\mathcal{P}_{H}^{r-1}$ the component-wise $L_{2}$-projection into $\left(\Pi^{r-1}\left(d_{H}\right)\right)^{N}$, let $\mathcal{G}_{H} u_{h}=\mathcal{P}_{H}^{r-1} \nabla u_{h}$.

Example 1.3. Let $d_{H}$ be as in Example 1.2 (except that the surface measure condition is now not needed), and set $\mathcal{G}_{H} v=\nabla \mathcal{P}_{H}^{r} v$. That is, in this case we first $L_{2}$-project into $\Pi^{r}\left(d_{H}\right)$ and then form the gradient. This may be thought of as a "continuous" version of Example 1.1. 
To relate our contribution to the vast literature on a posteriori error estimation, we shall next give a very brief (and not very exact) overview of relevant previous results.

The terminology "a posteriori" in numerical analysis seems to have originated in Ostrowski [8, p.111]. Referring in particular to the survey by Verfürth [10. Chapter 1], in finite element methods we have a posteriori error estimators of three different kinds: i) residual estimators; ii) local truncation error estimators, cf. also Babuška and Rheinboldt [1] and iii) averaging operator estimators, cf. also Zienkiewicz and Zhu [12]. Our Examples 1.1, 1.2 and 1.3 are of averaging type. In addition, we refer to Eriksson and Johnson [6] for an a posteriori estimator that is conceptually based on an a priori error estimate, and to Becker and Rannacher 3 for an estimator based on treating the Green's function as a weight; the latter approach is appropriate if the error needs to be assessed at a few points only. We also remark, see [10, p.19] for details, that local truncation error estimators may be interpreted as (approximately) lifting the residual from a dual space, and are thus related to residual estimators.

As seen in [10], under various conditions, certain local truncation error estimators and certain averaging operator estimators have been shown to be locally equivalent to residual estimators. A standard example of a residual estimator is the following: Let the equation to be solved be $-A u=f$, and let $\left[\partial v / \partial n_{A}\right]_{S}$ be the jump in the conormal derivative on an $(N-1)$-face $S$ of a simplex. Then set

$$
\mathcal{E}^{\mathrm{RES}}(\tau)=h\left\|A u_{h}+f\right\|_{L_{\infty}(\tau)}+\sum_{S \in \partial \tau}\left|\left[\frac{\partial u_{h}}{\partial n_{A}}\right]_{S}\right|_{L_{\infty}(S)} .
$$

Using a procedure of Verfürth (see [10, Proposition 1.5], and cf. Nochetto [7] and Dari, Durán and Padra [4] for the maximum-norm case), these can be shown to satisfy the following local efficiency property (modulo some "higher order" terms):

$$
C^{-1} \mathcal{E}^{\mathrm{RES}}(\tau) \leq\|\nabla e\|_{L_{\infty}(N(\tau))},
$$

where $N(\tau)=\bigcup\{\widetilde{\tau}: \widetilde{\tau}$ and $\tau$ have a common $(N-1)$-face $\}$. However, as for an upper (reliability) bound for $\nabla e$ in terms of $\mathcal{E}^{\mathrm{RES}}$ or other estimators which are locally equivalent with it as far as we know, for $N \geq 2$, previously only global upper bounds are known:

$$
\|\nabla e\|_{L_{\infty}(\Omega)} \leq C \max _{\tau} \mathcal{E}^{\mathrm{RES}}(\tau)
$$

(In fact, there are not even many such global bounds in maximum-norm, cf. [4] and [7, let alone for general space dimension $N>3$. Most a posteriori error investigations so far are done in the norms of $W_{2}^{1}(\Omega)$ or $L_{2}(\Omega)$, which are easier to handle than the pointwise norm. Even in those norms, the reliability bounds are global.)

On highly structured meshes one may have recourse to superconvergent points, say, points $\xi$ in an $O(h)$-neighborhood of $\tau$ for which one knows that, with $0<\delta \leq 1$,

$$
|\nabla e(\xi)| \leq C h^{r-1+\delta}\|u\|_{W_{\infty}^{r+1}(\Omega)} \quad \text { as } h \rightarrow 0 .
$$

One may then proceed e.g. as follows. Assuming that there are "enough" such points, interpolate (or, discretely $\ell_{2}$-project) to higher order at these points to create $\widetilde{\mathcal{G}}_{h} u_{h}$ such that also $\left\|\nabla u-\widetilde{\mathcal{G}}_{h} u_{h}\right\|_{L_{\infty}(\tau)} \leq C h^{r-1+\delta}\|u\|_{W_{\infty}^{r+1}(\Omega)}$, cf. e.g. Durán, Muschietti and Rodrigues [5]. Let $\widetilde{\mathcal{E}}(\tau)=\left\|\nabla u_{h}-\widetilde{\mathcal{G}}_{h} u_{h}\right\|_{L_{\infty}(\tau)}$ and, fixing $0<\varepsilon<1$, 
let $\widetilde{m}=C h^{\delta+\varepsilon-1}$. Suppose that (1.3) holds (Alternative I). Then

$$
\frac{1}{(1+\widetilde{m})} \widetilde{\mathcal{E}}(\tau) \leq\|\nabla e\|_{L_{\infty}(\tau)}
$$

and, if $\widetilde{m}<1$,

$$
\frac{1}{(1+\widetilde{m})} \widetilde{\mathcal{E}}(\tau) \leq\|\nabla e\|_{L_{\infty}(\tau)} \leq \frac{1}{(1-\widetilde{m})} \widetilde{\mathcal{E}}(\tau)
$$

In particular, if $\delta+\varepsilon>1$, then $\widetilde{\mathcal{E}}(\tau)$ is an asymptotically exact estimator. Suppose that (1.3) does not hold (Alternative II). Then

$$
\|\nabla e\|_{L_{\infty}(\tau)}+\widetilde{\mathcal{E}}(\tau) \leq(\widetilde{m}+C) h^{r-\varepsilon}\|u\|_{W_{\infty}^{r+1}(\Omega)}
$$

(see Corollary 2.3 below). We emphasize that, for $N \geq 2$ and simplicial meshes, a priori identifiable superconvergent points are known only on locally highly structured and regular meshes, cf. Wahlbin [1].

In brief then, we may sum up the contributions in the present investigation as follows: a) Local estimators are given which furnish both upper (reliability) and lower (efficiency) bounds for $\|\nabla e\|_{L_{\infty}(\tau)}$ on single elements; b) Asymptotically exact estimators are given on single elements without assuming any special structure of the mesh, beyond quasi-uniformity. We may also point out that we have not used so-called "saturation assumptions"; cf. [10, p.33] and Bank and Weiser [2, (2.5)], and the references therein.

It may seem paradoxical that we obtain such very local estimators by taking into account the values of $u_{h}$ on comparatively large neighborhoods of $\tau$. Our situation is somewhat related to the classical numerical analysis problem of optimizing $H$ in difference approximations $\partial_{H} v$ to $v^{\prime}$ when there are errors in the data $v$. In our context, we also have a "natural" approximation $v_{h}^{\prime}$ to $v^{\prime}$, and the question is to assess its accuracy. We assume that $\left|v-v_{h}\right| \leq h^{r}$ and $\left|\left(v-v_{h}\right)^{\prime}\right| \simeq h^{r-1}$, and take $\partial_{H} v(x)=H^{-1} \sum_{|j| \leq J} c_{j} v(x+j H)$ to be an $r$ th order approximation to $v^{\prime}$, with $\left|v^{\prime}-\partial_{H} v\right| \leq C H^{r}$ for $v$ smooth. We may assume that $\sum_{|j| \leq J}\left|c_{j}\right| \leq C$. Then

$$
\left|v^{\prime}-\partial_{H} v_{h}\right| \leq\left|v^{\prime}-\partial_{H} v\right|+\left|\partial_{H}\left(v-v_{h}\right)\right| \leq C\left(H^{r}+h^{r} / H\right) .
$$

Hence, if $\left|v^{\prime}-v_{h}^{\prime}\right| \geq h^{r-1}$ and $H$ is chosen so large that $C\left(H^{r}+h^{r} / H\right) \ll h^{r-1}$, then $\partial_{H} v_{h}$ is a better approximation to $v^{\prime}$ than $v_{h}^{\prime}$ is (and, in particular, $\left|v_{h}^{\prime}-\partial_{H} v_{h}\right| \simeq$ $\left.\left|\left(v-v_{h}\right)^{\prime}\right|\right)$. When viewed in this connection, averaging over a larger domain to obtain better local accuracy is less paradoxical. We remark that the contribution in the present paper consists to a large extent of making the above sketch rigorous.

In the rest of this paper we shall first give precise statements of our results (Section 2) and then prove them (Section 3). We conclude by verifying the assumptions (1.1) and (1.2) on $\mathcal{G}_{H}$ for the operators in Examples 1.1, 1.2 and 1.3 (Section 4).

\section{Precise statements of Results}

Let $\Omega$ be a bounded domain in $R^{N}$ with a sufficiently smooth boundary, and let

$$
A(v, w)=\int_{\Omega}\left(\sum_{i, j=1}^{N} a_{i j}(x) \frac{\partial v}{\partial x_{i}} \frac{\partial w}{\partial x_{j}}+\sum_{i=1}^{N} b_{i}(x) \frac{\partial v}{\partial x_{i}} w+c(x) v w\right) d x
$$


be a bilinear form on $W_{2}^{1}(\Omega) \times W_{2}^{1}(\Omega)$ with sufficiently smooth coefficients. Here, for any $\mathcal{D} \subseteq \Omega, 1 \leq p \leq \infty$, and $k=1,2,3, \ldots$, the space $W_{p}^{k}(\mathcal{D})$ is the Sobolev space with norm

$$
\|v\|_{W_{p}^{k}(\mathcal{D})}=\sum_{\ell=0}^{k}|v|_{W_{p}^{\ell}(\mathcal{D})}, \quad|v|_{W_{p}^{\ell}(\mathcal{D})}=\sum_{|\alpha|=\ell}\left\|D^{\alpha} v\right\|_{L_{p}(\mathcal{D})} .
$$

The form $A(\cdot, \cdot)$ is assumed to be uniformly elliptic, i.e., with $c_{\text {ell }}>0$,

$$
\sum_{i, j=1}^{N} \xi_{i} a_{i j}(x) \xi_{j} \geq c_{\mathrm{ell}}\left(\xi_{1}^{2}+\cdots+\xi_{N}^{2}\right) \quad \forall \xi \in R^{N}, \forall x \in \bar{\Omega},
$$

and it is also assumed to be coercive, $A(v, v) \geq c_{\text {coer }}\|v\|_{W_{2}^{1}(\Omega)}^{2} \forall v \in W_{2}^{1}(\Omega)$, with $c_{\text {coer }}>0$. In weak form, our problem is then to find $u \in W_{2}^{1}(\Omega)$ such that

$$
A(u, v)=\int_{\Omega} f v \quad \forall v \in W_{2}^{1}(\Omega),
$$

where $f$ is a given sufficiently smooth function. In second order partial differential equation form, the problem is

$$
-\sum_{i, j=1}^{N} \frac{\partial}{\partial x_{j}}\left(a_{i j} \frac{\partial u}{\partial x_{i}}\right)+\sum_{i=1}^{N} b_{i} \frac{\partial u}{\partial x_{i}}+c u=f \quad \text { in } \Omega,
$$

with the natural homogeneous conormal boundary condition

$$
\sum_{i=1}^{N} \frac{\partial u}{\partial x_{i}} \sum_{j=1}^{N} a_{i j} n_{j}=0 \quad \forall x \in \partial \Omega
$$

where $n_{j}=n_{j}(x)$ are the components of the unit normal to $\partial \Omega$.

For the finite element approximation of this problem, let $\mathcal{T}_{h}=\left\{\tau_{h}\right\}, 0<h<1$, be a sequence of triangulations of $\Omega, \bar{\Omega}=\bigcup_{\tau \in \mathcal{T}_{h}} \bar{\tau}$, with the elements $\tau$ mutually disjoint. Furthermore, we assume that each $\tau$ which does not meet $\partial \Omega$ is an $N$ dimensional straight simplex, while those $\tau$ that meet $\partial \Omega$ are allowed to have lowerdimensional curved faces in common with $\partial \Omega$. The partitions are face-to-face in that simplices meet only in full lower-dimensional faces or not at all. The triangulations are assumed to be globally quasi-uniform, i.e. (if necessary after a renormalization of $h$ ),

$$
\operatorname{diam} \tau \leq h \leq C_{q u}(\text { meas } \tau)^{1 / N} \quad \forall \tau \in \mathcal{T}_{h} .
$$

Our finite element spaces are then the $\mathcal{C}^{0}$ simplicial Lagrange elements

$$
S_{h}=S_{h}^{r-1}(\Omega)=\left\{\chi \in \mathcal{C}(\bar{\Omega}):\left.\chi\right|_{\tau} \in \Pi^{r-1}(\tau)\right\},
$$

where $\Pi^{r-1}(\mathcal{D})$ denotes the polynomials of total degree $\leq r-1$ on $\mathcal{D}$. Our approximation $u_{h} \in S_{h}$ to $u$ is defined by $A\left(u_{h}, \chi\right)=\int_{\Omega} f \chi, \forall \chi \in S_{h}$, or, equivalently, by $A\left(u_{h}-u, \chi\right)=0, \forall \chi \in S_{h}$.

For our gradient-like operator, let $d_{H}^{1} \subseteq d_{H}^{2}$ be two domains in $\Omega$ and let $\mathcal{G}_{H}$ : $\mathcal{C}\left(d_{H}^{2}\right) \rightarrow\left(\mathcal{C}\left(d_{H}^{1}\right)\right)^{N}$ be an operator such that

$$
\begin{gathered}
\operatorname{diam} d_{H}^{2} \leq H, \\
\left\|\mathcal{G}_{H} v\right\|_{L_{\infty}\left(d_{H}^{1}\right)} \leq C_{\mathcal{G}} H^{-1}\|v\|_{L_{\infty}\left(d_{H}^{2}\right)} \quad \forall v \in W_{\infty}^{1}\left(\bar{d}_{H}^{2}\right),
\end{gathered}
$$


and

$$
\left\|\nabla v-\mathcal{G}_{H} v\right\|_{L_{\infty}\left(d_{H}^{1}\right)} \leq C_{\mathcal{G}} H^{r}\|v\|_{W_{\infty}^{r+1}\left(d_{H}^{2}\right)} \quad \forall v \in \mathcal{C}^{r+1}\left(\bar{d}_{H}^{2}\right) .
$$

Let $\mathcal{T}_{h} \ni \tau \subseteq d_{H}^{1}$ and set

$$
\mathcal{E}(\tau)=\left\|\nabla u_{h}-\mathcal{G}_{H} u_{h}\right\|_{L_{\infty}(\tau)} .
$$

The following is the main result of this paper.

Theorem 2.1. Let $r \geq 3$ and fix $0<\varepsilon<1$. There exist two constants $C_{1}=$ $C_{1}\left(r, N, c_{\mathrm{ell}}, c_{\mathrm{coer}}, \Omega, a_{i j}, b_{i}, c, C_{\mathrm{qu}}, C_{\mathcal{G}}, \varepsilon\right)$ and $C_{2}$, which depends on the same quantities as $C_{1}$ except that it is independent of $\varepsilon$, such that, for $h$ small enough, for each $\tau \in \mathcal{T}_{h}$, we have one of the following two alternatives, where $m:=$ $C_{1}\left(h / H+(H / h)^{r} h^{\varepsilon}\right)$ and $u$ and $u_{h} \in S_{h}$ satisfy $A\left(u-u_{h}, \chi\right)=0, \forall \chi \in S_{h}$ :

Alternative I. Suppose that on the element $\tau$, the function enjoys the nondegeneracy condition

$$
|u|_{W_{\infty}^{r}(\tau)} \geq h^{1-\varepsilon}\|u\|_{W_{\infty}^{r+1}(\Omega)} .
$$

In this case

$$
\begin{gathered}
\left\|\nabla u-\mathcal{G}_{H} u_{h}\right\|_{L_{\infty}(\tau)} \leq m\|\nabla e\|_{L_{\infty}(\tau)}, \\
\frac{1}{(1+m)} \mathcal{E}(\tau) \leq\|\nabla e\|_{L_{\infty}(\tau)},
\end{gathered}
$$

and, if $m<1$,

$$
\frac{1}{(1+m)} \mathcal{E}(\tau) \leq\|\nabla e\|_{L_{\infty}(\tau)} \leq \frac{1}{(1-m)} \mathcal{E}(\tau)
$$

If $H=H(h)$ is chosen so that $m \rightarrow 0$ as $h \rightarrow 0$, the estimator is asymptotically exact.

Alternative II. Suppose (2.5) does not hold, i.e., $|u|_{W_{\infty}^{r}(\tau)}<h^{1-\varepsilon}\|u\|_{W_{\infty}^{r+1}(\Omega)}$. In this case

and

$$
\begin{gathered}
\|\nabla e\|_{L_{\infty}(\tau)} \leq C_{2} h^{r-\varepsilon}\|u\|_{W_{\infty}^{r+1}(\Omega)}, \\
\left\|\nabla u-\mathcal{G}_{H} u_{h}\right\|_{L_{\infty}(\tau)} \leq m h^{r-\varepsilon}\|u\|_{W_{\infty}^{r+1}(\Omega)},
\end{gathered}
$$

The results (2.7), (2.8), and (2.9), (2.11) are the main results for our a posteriori error estimators. In Alternative I, if $H=K h$ with $K$ sufficiently large, we have $m<1$ for $h$ sufficiently small, so that $\mathcal{E}(\tau)$ is an equivalent estimator. If $H=$ $H(h)$ is chosen so that $m=C_{2}\left(h / H+(H / h)^{r} h^{\varepsilon}\right) \rightarrow 0$ as $h \rightarrow 0$, then $\mathcal{E}(\tau)$ is asymptotically exact. Roughly speaking, this calls for $H$ being large compared to $h$ in a controlled fashion. The estimate (2.6), of which (2.7) and (2.8) are trivial consequences via the triangle inequality since $\nabla u_{h}-\mathcal{G}_{H} u_{h}=-\nabla e+\left(\nabla u-\mathcal{G}_{H} u\right)$, hints at a fundamental underlying reason why our a posteriori estimators work: for $m<1, \mathcal{G}_{H} u_{h}$ is a "better" approximation of $\nabla u$ than is $\nabla u_{h}$ itself. (The role of (2.10) in Alternative II is less clear.)

To elucidate the relations between $|u|_{W_{\infty}^{r}(\tau)}$ and $h^{1-\varepsilon}\|u\|_{W_{\infty}^{r+1}(\Omega)}$ in Alternatives I and II, we give the following corollary of the proof of the theorem. 
Corollary 2.2. i) If (2.5) holds, then

$$
C_{2}^{-1} h^{r-1}|u|_{W_{\infty}^{r}(\tau)} \leq\|\nabla e\|_{L_{\infty}(\tau)} \leq C_{2} h^{r-1}|u|_{W_{\infty}^{r}(\tau)} \text {. }
$$

ii) If (2.5) holds, then

$$
\|\nabla e\|_{L_{\infty}(\tau)} \geq C_{2}^{-1} h^{r-\varepsilon}\|u\|_{W_{\infty}^{r+1}(\Omega)} .
$$

Conversely, if $\|\nabla e\|_{L_{\infty}(\tau)} \geq C_{2} h^{r-\varepsilon}\|u\|_{W_{\infty}^{r+1}(\Omega)}$, then (2.5) holds.

In other words, the condition in Alternative I implies that

$$
\|\nabla e\|_{L_{\infty}(\tau)} \simeq h^{r-1}|u|_{W_{\infty}^{r}(\tau)},
$$

and it is "equivalent" to

$$
\|\nabla e\|_{L_{\infty}(\tau)} \gtrsim h^{r-\varepsilon}\|u\|_{W_{\infty}^{r+1}(\Omega)} .
$$

The equivalence (2.12) says that $\nabla e$ behaves on $\tau$ exactly as one would expect from elementary approximation theory. Indeed, the lower bound follows from elementary approximation theory, but the upper bound requires a sharply localized a priori estimate from [9]. One may also interpret (2.12) as saying that there is no "pollution" into $\tau$ from outside. (We shall treat nonsmooth problems in a future paper.)

Finally, we shall state the result on the use of superconvergence in a posteriori error estimation mentioned in the Introduction.

Corollary 2.3. Assume that $\widetilde{\mathcal{G}}_{h}$ is an operator such that, with $\delta>0$,

$$
\left\|\nabla u-\widetilde{\mathcal{G}}_{h} u_{h}\right\|_{L_{\infty}(\tau)} \leq C_{\widetilde{\mathcal{G}}} h^{r-1+\delta}\|u\|_{W_{\infty}^{r+1}(\Omega)} .
$$

Fix $0<\varepsilon<1$. There exists then a constant $\widetilde{C}$ such that, with $\widetilde{m}=\widetilde{C} h^{\delta+\varepsilon-1}$, we have the following assertions for $\widetilde{\mathcal{E}}(\tau)=\left\|\nabla u_{h}-\widetilde{\mathcal{G}}_{h} u_{h}\right\|_{L_{\infty}(\tau)}$ :

i) If (2.5) holds, then

$$
\frac{1}{(1+\widetilde{m})} \widetilde{\mathcal{E}}(\tau) \leq\|\nabla e\|_{L_{\infty}(\tau)},
$$

and further, if $\widetilde{m}<1$,

$$
\frac{1}{(1+\widetilde{m})} \widetilde{\mathcal{E}}(\tau) \leq\|\nabla e\|_{L_{\infty}(\tau)} \leq \frac{1}{(1-\widetilde{m})} \widetilde{\mathcal{E}}(\tau) .
$$

In particular, if $\delta+\varepsilon>1$, the estimator is asymptotically exact as $h \rightarrow 0$.

ii) If (2.5) does not hold, then

$$
\|\nabla e\|_{L_{\infty}(\tau)}+\widetilde{\mathcal{E}}(\tau) \leq(\widetilde{m}+\widetilde{C}) h^{r-\varepsilon}\|u\|_{W_{\infty}^{r+1}(\Omega)} .
$$

We remark that an operator $\widetilde{\mathcal{G}}_{h}$ satisfying $(2.13)$ can be constructed not only when there are natural superconvergent points for $\nabla e$,

$$
|\nabla e(\xi)| \leq C h^{r-1+\delta}\|u\|_{W_{\infty}^{r+1}(\Omega)},
$$

but also when superconvergence occurs in function values,

$$
|e(\eta)| \leq C h^{r+\delta}\|u\|_{W_{\infty}^{r+1}(\Omega)} .
$$

Indeed, this pointwise superconvergence may itself have been attained by postprocessing with an operator $G_{h}$ so that

$$
\left|\left(\nabla u-G_{h} u_{h}\right)(\xi)\right| \leq C h^{r-1+\delta}\|u\|_{W_{\infty}^{r+1}(\Omega)},
$$

and similarly for function values. 


\section{Proof of Theorem 2.1}

We shall first state a proposition which will imply Theorem 2.1 by elementary considerations. The constants involved in that proposition and the theorem are slight variations of one another and, for clarity, we denote them $\widehat{C}_{1}$ and $\widehat{C}_{2}$ below, and also set $\widehat{m}=\widehat{C}_{1}\left(h / H+(H / h)^{r} h^{\varepsilon}\right)$. In the proposition, there are no relations imposed between $|u|_{W_{\infty}^{r}(\tau)}$ and $h^{1-\varepsilon}\|u\|_{W_{\infty}^{r+1}(\Omega)}$ or between $H$ and $h$ (except, of course, $H \geq h$ ).

Proposition 3.1. Let the general assumptions of Theorem 2.1 hold, and let $H \geq h$. Then

$$
\left\|\nabla u-\mathcal{G}_{H} u_{h}\right\|_{L_{\infty}(\tau)} \leq \widehat{m}\left(h^{r-1}|u|_{W_{\infty}^{r}(\tau)}+h^{r-\varepsilon}\|u\|_{W_{\infty}^{r+1}(\Omega)}\right)
$$

and

$$
\begin{gathered}
\widehat{C}_{2}^{-1}\left(h^{r-1}|u|_{W_{\infty}^{r}(\tau)}-h^{r}\|u\|_{W_{\infty}^{r+1}(\Omega)}\right) \leq\|\nabla e\|_{L_{\infty}(\tau)} \\
\leq \widehat{C}_{2}\left(h^{r-1}|u|_{W_{\infty}^{r}(\tau)}+h^{r}\|u\|_{W_{\infty}^{r+1}(\Omega)}\right) .
\end{gathered}
$$

To see how Theorem 2.1 follows from this, for definiteness, let us take $h$ small enough so that $h^{\varepsilon} \leq 1 / 2$. In the case of Alternative I, we have, from (3.1),

$$
\left\|\nabla u-\mathcal{G}_{H} u_{h}\right\|_{L_{\infty}(\tau)} \leq 2 \widehat{m} h^{r-1}|u|_{W_{\infty}^{r}(\tau)}
$$

and, from the first part of (3.2),

$$
\begin{aligned}
& \frac{1}{2} \widehat{C}_{2}^{-1} h^{r-1}|u|_{W_{\infty}^{r}(\tau)} \leq \widehat{C}_{2}^{-1}\left(1-h^{\varepsilon}\right) h^{r-1}|u|_{W_{\infty}^{r}(\tau)} \\
& \quad \leq \widehat{C}_{2}^{-1}\left(h^{r-1}|u|_{W_{\infty}^{r}(\tau)}-h^{r}\|u\|_{W_{\infty}^{r+1}(\Omega)}\right) \leq\|\nabla e\|_{L_{\infty}(\tau)},
\end{aligned}
$$

so that

$$
\left\|\nabla u-\mathcal{G}_{H} u_{h}\right\|_{L_{\infty}(\tau)} \leq 4 \widehat{C}_{2} \widehat{m}\|\nabla e\|_{L_{\infty}(\tau)} .
$$

Thus, (2.6) follows with $C_{1}=4 \widehat{C}_{2} \widehat{C}_{1}$. As already noted, (2.7) and (2.8) are trivial consequences of (2.6) and the triangle inequality.

In Alternative II, from the second part of (3.2) we have

$$
\|\nabla e\|_{L_{\infty}(\tau)} \leq \widehat{C}_{2}\left(1+h^{\varepsilon}\right) h^{r-\varepsilon}\|u\|_{W_{\infty}^{r+1}(\Omega)} \leq 3 \widehat{C}_{2} / 2 h^{r-\varepsilon}\|u\|_{W_{\infty}^{r+1}(\Omega)},
$$

and (2.9) follows with $C_{2}=3 \widehat{C}_{2} / 2$. As for (2.10),

$$
\left\|\nabla u-\mathcal{G}_{H} u_{h}\right\|_{L_{\infty}(\tau)} \leq 2 \widehat{m} h^{r-\varepsilon}\|u\|_{W_{\infty}^{r+1}(\Omega)},
$$

and (2.10) follows with $C_{1}=2 \widehat{C}_{1}$. Taking $C_{1}=\max \left(4 \widehat{C}_{2} \widehat{C}_{1}, 2 \widehat{C}_{1}\right)$ and $C_{2}=3 \widehat{C}_{2} / 2$ gives us Theorem 2.1 (since (2.11) follows from (2.9) and (2.10) by the triangle inequality).

Corollaries 2.2 and 2.3 follow in similar elementary fashions: Corollary 2.2 uses (3.2) alone, and Corollary 2.3 uses (3.2) and (2.13). We shall not give the simple details.

The rest of this section will be concerned with the proof of Proposition 3.1. The following results are fundamental technical tools in the proof.

Lemma 3.2. Let $r \geq 3$ and $0<\varepsilon^{\prime}<1$. For any $x \in \Omega$,

$$
|e(x)| \leq C_{\varepsilon^{\prime}} h^{r}\left(\sum_{|\alpha|=r}\left|D^{\alpha} u(x)\right|+h^{1-\varepsilon^{\prime}}\|u\|_{W_{\infty}^{r+1}(\Omega)}\right)
$$


and

$$
|\nabla e(x)| \leq C h^{r-1}\left(\sum_{|\alpha|=r}\left|D^{\alpha} u(x)\right|+h\|u\|_{W_{\infty}^{r+1}(\Omega)}\right) .
$$

The proofs are given in 9, Theorem 4.1 with $t=r+1-\varepsilon^{\prime}$ and Theorem 4.2 with $t=r$, respectively]. We remark that, for $r \geq 4$, we may take $\varepsilon^{\prime}=0$ also in (3.3).

The second inequality in (3.2) is immediate from (3.4). For the first inequality we have

Lemma 3.3. The first inequality in (3.2) holds, i.e.,

$$
\widehat{C}_{2}^{-1}\left(h^{r-1}|u|_{W_{\infty}^{r}(\tau)}-h^{r}\|u\|_{W_{\infty}^{r+1}(\Omega)}\right) \leq\|\nabla e\|_{L_{\infty}(\tau)} .
$$

Proof. If $|u|_{W_{\infty}^{r}(\tau)}=0$ there is nothing to prove. Hence we may assume that $|u|_{W^{r}(\tau)} \neq 0$, and there exist a multi-index $\beta_{0}$ with $\left|\beta_{0}\right|=r$, and a point $y_{0} \in \bar{\tau}$ such that for some $\bar{c}>0$

$$
\left|D^{\beta_{0}} u\left(y_{0}\right)\right| \geq \bar{c}|u|_{W_{\infty}^{r}(\tau)} .
$$

Suppose that $\beta_{0 i} \neq 0$; then

$$
\begin{aligned}
\min _{\chi \in \Pi^{r-1}}\|\nabla(u-\chi)\|_{L_{\infty}(\tau)} & \geq \min _{\chi \in \Pi^{r-1}}\left\|\frac{\partial u}{\partial x_{i}}-\frac{\partial \chi}{\partial x_{i}}\right\|_{L_{\infty}(\tau)} \\
& \geq \min _{\psi \in \Pi^{r-2}}\left\|\frac{\partial u}{\partial x_{i}}-\psi\right\|_{L_{\infty}(\tau)} .
\end{aligned}
$$

By Taylor's theorem, we have for any $x \in \bar{\tau}$

$$
\frac{\partial u}{\partial x_{i}}(x)=\sum_{|\alpha| \leq r-1} \frac{1}{\alpha !}\left(D^{\alpha} \frac{\partial u}{\partial x_{i}}\right)\left(y_{0}\right)\left(x-y_{0}\right)^{\alpha}+R,
$$

where $R$ is the remainder

$$
R=r \sum_{|\alpha|=r}\left(x-y_{0}\right)^{\alpha} \int_{0}^{1} \frac{1}{\alpha !} s^{r-1}\left(D^{\alpha} \frac{\partial u}{\partial x_{i}}\right)\left(x+s\left(y_{0}-x\right)\right) d s,
$$

and

$$
\|R\|_{L_{\infty}(\tau)} \leq \widehat{C} h^{r}|u|_{W_{\infty}^{r+1}(\tau)} .
$$

Let $\widehat{\beta}_{0}=\left(\beta_{01}, \ldots, \beta_{0 i}-1, \ldots, \beta_{0 N}\right)$ and let $\widehat{\Pi}^{r-1}$ denote the set of polynomials of degree $\leq r-1$ of the form $\sum_{|\alpha| \leq r-1} k_{\alpha}\left(x-y_{0}\right)^{\alpha}$ with $k_{\hat{\beta}_{0}}=0$. Thus,

$$
\sum_{|\alpha| \leq r-1} \frac{1}{\alpha !}\left(D^{\alpha} \frac{\partial u}{\partial x_{i}}\right)\left(y_{0}\right)\left(x-y_{0}\right)^{\alpha}=\widehat{\psi}+\frac{1}{\widehat{\beta}_{0} !}\left(D^{\beta_{0}} u\right)\left(y_{0}\right)\left(x-y_{0}\right)^{\hat{\beta}_{0}}, \widehat{\psi} \in \widehat{\Pi}^{r-1} .
$$

Using the triangle inequality, (3.7), and (3.8),

$$
\begin{aligned}
\min _{\psi \in \Pi^{r-2}} & \left\|\frac{\partial u}{\partial x_{i}}-\psi\right\|_{L_{\infty}(\tau)} \\
& \geq \min _{\psi \in \widehat{\Pi}^{r-1}}\left\|\widehat{\psi}+\frac{1}{\widehat{\beta}_{0} !} D^{\beta_{0}} u\left(y_{0}\right)\left(x-y_{0}\right)^{\widehat{\beta}_{0}}-\psi\right\|_{L_{\infty}(\tau)}-\|R\|_{L_{\infty}(\tau)} \\
& \geq \min _{\varphi \in \widehat{\Pi}^{r-1}}\left\|\frac{1}{\widehat{\beta}_{0} !} D^{\beta_{0}} u\left(y_{0}\right)\left(x-y_{0}\right)^{\widehat{\beta}_{0}}-\varphi\right\|_{L_{\infty}(\tau)}-\widehat{C} h^{r}\|u\|_{W_{\infty}^{r+1}(\tau)} .
\end{aligned}
$$


Since $\left(x-y_{0}\right)^{\hat{\beta}_{0}} \notin \widehat{\Pi}^{r-1}$, it follows by scaling and by (3.5) that for some $c>0$ independent of $u$ and $h$,

$$
\min _{\varphi \in \widehat{\Pi}^{r-1}}\left\|\frac{1}{\widehat{\beta}_{0} !} D^{\beta_{0}} u\left(y_{0}\right)\left(x-y_{0}\right)^{\hat{\beta}_{0}}-\varphi\right\|_{L_{\infty}(\tau)} \geq c h^{r-1}|u|_{W_{\infty}^{r}(\tau)} .
$$

The lemma now follows from (3.6), (3.9) and (3.10).

We have thus proven (3.2).

To complete the proof of Proposition 3.1, it now remains to prove (3.1). From the triangle inequality, (2.3) and (2.2) we have

$$
\begin{aligned}
\left\|\nabla u-\mathcal{G}_{H} u_{h}\right\|_{L_{\infty}(\tau)} & \leq\left\|\nabla u-\mathcal{G}_{H} u\right\|_{L_{\infty}(\tau)}+\left\|\mathcal{G}_{H} e\right\|_{L_{\infty}(\tau)} \\
& \leq C_{\mathcal{G}}\left(H^{r}\|u\|_{W_{\infty}^{r+1}(\Omega)}+H^{-1}\|e\|_{L_{\infty}\left(d_{H}^{2}\right)}\right) .
\end{aligned}
$$

Let $\|e\|_{L_{\infty}\left(d_{H}^{2}\right)}=|e(\bar{x})|$. From (3.3) of Lemma 3.2, we have, with $\varepsilon^{\prime}=\varepsilon$, since $\operatorname{dist}(\bar{x}, \tau) \leq H$,

$$
\begin{aligned}
|e(\bar{x})| & \leq C_{\varepsilon} h^{r}\left(\sum_{|\alpha|=r}\left|D^{\alpha} u(\bar{x})\right|+h^{1-\varepsilon}\|u\|_{W_{\infty}^{r+1}(\Omega)}\right) \\
& \leq C_{\varepsilon} h^{r}\left(|u|_{W_{\infty}^{r}(\tau)}+\left(H+h^{1-\varepsilon}\right)\|u\|_{W_{\infty}^{r+1}(\Omega)}\right) .
\end{aligned}
$$

Thus, from (3.11), using that $h^{r} \leq H^{r}$,

$$
\begin{aligned}
\left\|\nabla u-\mathcal{G}_{H} u_{h}\right\|_{L_{\infty}(\tau)} & \leq C\left(\frac{h^{r}}{H}|u|_{W_{\infty}^{r}(\tau)}+\left(H^{r}+\frac{h^{r+1-\varepsilon}}{H}\right)\|u\|_{W_{\infty}^{r+1}(\Omega)}\right) \\
& =C\left(h^{r-1}|u|_{W_{\infty}^{r}(\tau)}\left(\frac{h}{H}\right)+h^{r-\varepsilon}\|u\|_{W_{\infty}^{r+1}(\Omega)}\left(\left(\frac{H}{h}\right)^{r} h^{\varepsilon}+\frac{h}{H}\right)\right),
\end{aligned}
$$

which proves (3.1).

This completes the proof of Proposition 3.1 and hence of Theorem 2.1.

4. Verification of (2.2) ANd (2.3) in Examples 1.1, 1.2 AND 1.3

All these properties are standard except possibly (2.2) in the context of Example 1.2 .

Example 1.1. Recall that here $d_{H}$ is a shape-regular simplex of diameter $H$, and $\mathcal{G}_{H} v=\nabla I_{H}^{r} v$. In the case of $d_{H}$ abutting on $\partial \Omega$, if $d_{H}$ has a curved face, we use interpolation to a slightly smaller but still shape-regular straight simplex. Since pointwise interpolation at the principal lattice points into $\Pi^{r}\left(d_{H}\right)$ is a bounded operation on $\mathcal{C}\left(\bar{d}_{H}\right)$, the property $(2.2)$ is simply an inverse inequality. The property (2.3) is a standard approximation result.

Example 1.2. Under our assumptions with respect to the balls $\underline{B}$ and $\bar{B}$, we have, as is well known, the following inverse inequalities for $\chi \in \Pi^{r-1}\left(d_{H}\right)$ :

$$
\begin{gathered}
\|\chi\|_{L_{p}\left(d_{H}\right)} \leq C H^{-N\left(\frac{1}{q}-\frac{1}{p}\right)}\|\chi\|_{L_{q}\left(d_{H}\right)}, 1 \leq p, q \leq \infty, \\
\|\nabla \chi\|_{L_{p}\left(d_{H}\right)} \leq C H^{-1}\|\chi\|_{L_{p}\left(d_{H}\right)}, 1 \leq p \leq \infty .
\end{gathered}
$$

Let us now consider the $L_{2}$-projection $\mathcal{P}_{H}^{r-1} w$ for $w$ a scalar function.

Lemma 4.1. There exists a constant $C$ such that

$$
\left\|\mathcal{P}_{H}^{r-1} w\right\|_{L_{p}\left(d_{H}\right)} \leq C\|w\|_{L_{p}\left(d_{H}\right)}, 1 \leq p \leq \infty .
$$


Proof. The estimate is trivial when $p=2$, in which case $C=1$. For $p>2$, we have, using (4.1),

$$
\begin{aligned}
\left\|\mathcal{P}_{H}^{r-1} w\right\|_{L_{p}\left(d_{H}\right)} & \leq C H^{-N\left(\frac{1}{2}-\frac{1}{p}\right)}\left\|\mathcal{P}_{H}^{r-1} w\right\|_{L_{2}\left(d_{H}\right)} \\
& \leq C H^{-N\left(\frac{1}{2}-\frac{1}{p}\right)}\|w\|_{L_{2}\left(d_{H}\right)} \leq C\|w\|_{L_{p}\left(d_{H}\right)},
\end{aligned}
$$

where we used Hölder's inequality in the last step.

For $p<2$, we use a duality argument

$$
\left\|\mathcal{P}_{H}^{r-1} w\right\|_{L_{p}\left(d_{H}\right)}=\sup _{\|\eta\|_{L_{q}\left(d_{H}\right)}=1} \int_{d_{H}}\left(\mathcal{P}_{H}^{r-1} w\right) \eta
$$

where $1 / p+1 / q=1$. For each such $\eta$, we have, since $q>2$,

$$
\int_{d_{H}}\left(\mathcal{P}_{H}^{r-1} w\right) \eta=\int_{d_{H}} w\left(\mathcal{P}_{H}^{r-1} \eta\right) \leq\|w\|_{L_{p}\left(d_{H}\right)}\left\|\mathcal{P}_{H}^{r-1} \eta\right\|_{L_{q}\left(d_{H}\right)} \leq C\|w\|_{L_{p}\left(d_{H}\right)} .
$$

This proves the lemma.

We now consider the properties (2.2) and (2.3). We start with (2.3). Let $d_{H}^{1}=$ $d_{H}, d_{H}^{2}=\bar{B} \cap \Omega$. Using a Taylor polynomial $T v$ of degree $r$ centered at $\bar{x}$, we have $\mathcal{G}_{H} v-v=\left(\mathcal{P}_{H}^{r-1}-I\right) \nabla(v-T v)$. By (4.3) with $p=\infty,(2.3)$ is immediate, since $d_{H}^{2}$ is star-shaped with respect to $\bar{x}$.

Next consider (2.2). Here we shall use a duality argument. Consider $\mathcal{P}_{H}^{r-1} \frac{\partial v}{\partial x_{i}}$, $i=1, \ldots, N$. Then

$$
\left\|\mathcal{P}_{H}^{r-1} \frac{\partial v}{\partial x_{i}}\right\|_{L_{\infty}\left(d_{H}\right)}=\sup _{\|\eta\|_{L_{1}\left(d_{H}\right)}=1} \int_{d_{H}}\left(\mathcal{P}_{H}^{r-1} \frac{\partial v}{\partial x_{i}}\right) \eta .
$$

For each such $\eta$, by integration by parts,

$$
\begin{aligned}
\int_{d_{H}}\left(\mathcal{P}_{H}^{r-1} \frac{\partial v}{\partial x_{i}}\right) \eta & =\int_{d_{H}} \frac{\partial v}{\partial x_{i}}\left(\mathcal{P}_{H}^{r-1} \eta\right) \\
& =\oint_{\partial d_{H}} v\left(\mathcal{P}_{H}^{r-1} \eta\right) n_{i} d \sigma-\int_{d_{H}} v \frac{\partial}{\partial x_{i}}\left(\mathcal{P}_{H}^{r-1} \eta\right)=I_{1}+I_{2}
\end{aligned}
$$

where $n_{i}$ is the $i$ th component of the outward unit normal for $d_{H}$. We have

$$
\begin{aligned}
\left|I_{1}\right| & \leq\|v\|_{L_{\infty}\left(d_{H}\right)}\left\|\mathcal{P}_{H}^{r-1} \eta\right\|_{L_{\infty}\left(d_{H}\right)} \operatorname{meas}_{N-1}\left(\partial d_{H}\right) \\
& \leq C\|v\|_{L_{\infty}\left(d_{H}\right)} H^{-N}\left\|\mathcal{P}_{H}^{r-1} \eta\right\|_{L_{1}\left(d_{H}\right)} H^{N-1}
\end{aligned}
$$

where we used the inverse estimate (4.1) $(p=\infty, q=1)$ and our assumption on the surface measure of $\partial d_{H}$. From Lemma 4.1 (with $p=1$ ) we thus obtain

$$
\left|I_{1}\right| \leq C H^{-1}\|v\|_{L_{\infty}\left(d_{H}\right)} \text {. }
$$

For $I_{2}$ we have, using the inverse estimate for gradients (4.2) with $p=1$ and again Lemma 4.1 with $p=1$,

$$
\left|I_{2}\right| \leq\|v\|_{L_{\infty}\left(d_{H}\right)}\left\|\frac{\partial}{\partial x_{i}} \mathcal{P}_{H}^{r-1} \eta\right\|_{L_{1}\left(d_{H}\right)} \leq C H^{-1}\|v\|_{L_{\infty}\left(d_{H}\right)} .
$$

Together, (4.4) and (4.5) show that

$$
\left\|\mathcal{G}_{H} v\right\|_{L_{\infty}\left(d_{H}\right)} \leq C H^{-1}\|v\|_{L_{\infty}\left(d_{H}\right)} .
$$

Example 1.3. As in the previous example, $\mathcal{P}_{H}^{r}$ is stable in $L_{\infty}\left(d_{H}\right)$. Condition (2.2) then follows from (4.2), and (2.3) again follows by use of the Taylor polynomial centered at $\bar{x}$. 


\section{ACKNOWLEDGMENT}

We thank an anonymous referee for several interesting comments.

\section{REFERENCES}

1. I. Babuška and W. Rheinboldt, A posteriori error estimates for the finite element method, Internat. J. Numer. Methods Engrg. 12 (1978), 1597-1615.

2. R. E. Bank and A. Weiser, Some a posteriori error estimators for elliptic partial differential equations, Math. Comp. 44 (1985), 283-301. MR 86g:65207

3. R. Becker and R. Rannacher, Weighted a posteriori error control in finite element methods, ENUMATH-95, Paris, 1995, in Proceedings ENUMATH-97, H. G. Brock et al., eds., World Scientific, River Edge, NJ, 1998, pp. 621-637.

4. E. A. Dari, R. Durán, and C. Padra, Maximum norm error estimators for three dimensional elliptic problems, SIAM J. Numer. Anal. 37 (2000), 683-700. CMP 2000:08

5. R. Durán, M. A. Muschietti and R. Rodriguez, On the asymptotic exactness of error estimators for linear triangular elements, Numer. Math. 59 (1991), 107-127. MR 92b:65086

6. K. Eriksson and C. Johnson, An adaptive finite element method for linear elliptic problems, Math. Comp. 50 (1988), 361-383. MR 89c:65119

7. R. H. Nochetto, Pointwise a posteriori estimates for elliptic problems on highly graded meshes, Math. Comp. 64 (1995), 1-22. MR 95c:65172

8. A. Ostrowski, Recherches sur la méthode de Graeffe et les zéros de polynomes et des séries de Laurent, Acta Math. 72 (1940), 99-257. MR 1:323f MR 2:342c

9. A. H. Schatz, Pointwise error estimates and asymptotic error expansion inequalities for the finite element method on irregular grids: Part 1, Global estimates, Math. Comp. 67 (1998), 877-899. MR 98j:65082

10. R. Verfürth, A Review of A Posteriori Error Estimation and Adaptive Mesh-Refinement Techniques, Wiley and Teubner, 1996.

11. L. B. Wahlbin, Superconvergence in Galerkin Finite Element Methods, Lecture Notes in Mathematics 1605, Springer, 1995. MR 98j:65083

12. O. C. Zienkiewicz and J. Z. Zhu, The superconvergent patch recovery and a posteriori error estimates, Parts 1 and 2, Internat. J. Numer. Methods Engrg. 33 (1992), 1331-1364 and 1365-382. MR 93c:73098: MR 93c:73099

ICA 3, Universität Stuttgart, Pfaffenwaldring 27, Stuttgart, Germany

E-mail address: wolfgang@ica3.uni-stuttgart.de

Department of Mathematics, Cornell University, Ithaca, New York 14853

E-mail address: schatz@math.cornell.edu

Department of Mathematics, Cornell University, Ithaca, New York 14853

E-mail address: wahlbin@math.cornell.edu

IWR, Universität Heidelberg, in Neuenheimer Feld 368, Heidelberg, Germany

E-mail address: wittum@iwr.uni-heidelberg.de 